\title{
On metamorphosis of Kummer surfaces
}

\author{
Isao NARUKI \\ (Received October 29, 1990, Revised March 11, 1991)
}

\section{Introduction}

One method of introducing $K 3$ surfaces with large Picard number is to create degenerate elements having at most rational double points in some fixed category of $K 3$ surfaces (e.g. quartic surfaces in $\boldsymbol{P}^{3}$, sextic double planes etc. See Brieskorn [2][3]). Then we are sometimes led to a situation such that, for a surface obtained in this way, a lattice isomorphic to $L\left(E_{8}\right) \oplus N$ is primitively imbedded into its Néron-Severi group. Under the algebraicity assumption, D. Morrison proved that such a surface is a Kummer surface and vice versa [6]. $\left(L\left(E_{8}\right)\right.$ denotes the root lattice of the simple group $E_{8}$, and $N$ denotes the Nikulin lattice. $N$ is given by the canonical $\boldsymbol{Q}$-basis $\left\{e_{1}, e_{2}, \ldots, e_{8}\right\}$ such that $\left(e_{i}, e_{j}\right)=-2 \delta_{i j}$ and that $e_{1}, \ldots, e_{8}$, $\sum_{i=1}^{\infty} e_{i} / 2$ generate $N$ over $\boldsymbol{Z}$.) However, Morrison's proof is numbertheoretic and it is in general an extremely difficult problem to find sixteen disjoint non-singular rational curves which should exist on the surface. In this note we will give a solution to this problem in the case when the Néron-Severi lattice is of rank 17 and of determinant $2^{6}$ i. e. in the case corresponding to (generic) Kummer quartics. We will namely describe the inverse process explicitly by finding a lattice isomorphic to $L\left(E_{8}\right) \oplus N$ in the Néron-Severi group of the Kummer quartic. The lattice to be found should be effective in the sense that it has the canonical base $\left\{r_{1}, r_{2}\right.$, $\left.\ldots, r_{8}, e_{1}, e_{2}, \ldots, e_{8}\right\}$, represented by non-singular rational curves such that $r_{1}, r_{2}, \ldots, r_{8}$ form a Dynkin base of $L\left(E_{8}\right)$, and that $\left\{e_{1}, e_{2}, \ldots, e_{8}\right\}$ is the canonical $\boldsymbol{Q}$-base of $N$. (The genericity assumption is imposed exactly to guarantee this effectiveness.) It is now clear how to recover the classes of sixteen exceptional curves from the canonical basis $\left\{r_{i}, e_{i}\right\}$ when the latter is known in the geometric context. This is of great importance since it enables us to describe the parameter space of the $K 3$ surface, which is theoretically known to be a Kummer quartic, by the theta constants; they are in principle calculated from the Kummer configuration in $P^{3}(\boldsymbol{C})$ consisting of sixteen singular points and sixteen singular planes by the classical works of Borchardt [1], Cayley [4], Weber [8] and Humbert [5]. But we will not be concerned with such a detailed computation in this note; we will only show the equivalence between the Kummer quartics and some 
special kind of sextic double planes to clarify this phenomenology about Kummer surfaces.

\section{$\S 1$. Kummer configuration}

In this section we will briefly study the classical Kummer configuration as a preliminary to the construction of the lattice $L\left(E_{8}\right) \oplus N$. Suppose that $S$ is a quartic surface in $P^{3}(\boldsymbol{C})$ with sixteen ordinary double points. The argument in this section is not completely intrinsic, but it only depends on one double point on $S$ chosen as the source of projection; we denote it by $q_{0}$. To be precise with the notion of the projection through $q_{0}$, we form the blowing up $\widehat{P^{3}(\boldsymbol{C})}$ of $P^{3}(\boldsymbol{C})$ with center $q_{0}$, by which the partial desingularization at $q_{0}$ is the proper transform of $S$ to $\widehat{P^{3}(\boldsymbol{C})}$. We denote this by $S_{0}: S_{0} \hookrightarrow \widehat{P^{3}(\boldsymbol{C})} . \widehat{P^{3}(\boldsymbol{C})}$ is a $P^{1}(\boldsymbol{C})$-bundle over the exceptional set (in the space) which is isomorphic to $P^{2}(\boldsymbol{C})$. By restricting the canonical projection to $S_{0}$ we regard $S_{0}$ as a double cover of this $P^{2}(\boldsymbol{C})$. The branch locus in sextic curve with fifteen nodes corresponding to the remaining double points. Such a sextic is automatically decomposed into 6 lines intersecting transversally. We index the lines to be $l_{1}, l_{2}, \ldots, l_{6}$. The image on $P^{2}(C)$ of the exceptional curve of the partial desingularization $S_{0} \rightarrow S$ is a conic and each $l_{i}$ is tangent to the conic. By $p_{j k}(1 \leq j<k \leq 6)$ we denote the intersection point of $l_{j}$ and $l_{k}$, and by $q_{j k}$ the singular point of $S_{0}$ (and of $S$ ) lying over $p_{j k}$. We have the singular locus :

$$
\Sigma(S)=\left\{q_{0}, q_{12}, q_{13}, \ldots, q_{56}\right\} .
$$

The inverse image of $l_{i}$ in $\widehat{P^{3}(\boldsymbol{C})}$ is a proper transform of a plane in $P^{3}(\boldsymbol{C})$, which we will denote by $H_{i}$. The intersection $H_{i} \cap S$ is a (doubly counted) conic in $H_{i}$; it is naturally isomorphic to the line $l_{i}$ by the projection $S_{0} \rightarrow P^{2}(\boldsymbol{C})$ when lifted to $S_{0} . H_{i}$ is a special plane for $S$ on which exactly six singular points lie; those are $q_{0}, q_{1 i}, \ldots, q_{(i-1) i}, q_{i(i+1)}, \ldots, q_{i 6}$. Such a plane is called a singular plane of the Kummer quartic $S$. Since $q_{0}$ is not a special double point, we have seen that, for each double point, there are six singular planes passing through it. We have therefore sixteen singular planes to sum up. Now the question arises: How can the remaining ten be described by the geometry of the double plane $S_{0} \rightarrow$ $P^{2}(\boldsymbol{C})$ ? On each of them there are six singular points and they lie on the conic which is the intersection with $S_{0}$. Thus, by the projection with source $q_{0}$, they are in a one-to-one correspondence with the conconical 
sixtuplets out of the set of points $p_{j k}, L \leq j<k \leq 6$. These ten sixtuplets are listed in the following way: Suppose we are given a $(3,3)$-partition of the index set $\{1,2, \ldots, 6\}$, say $\{i<j<k, l<m<n\}$. Then $\left\{p_{i j}, p_{i k}, p_{j k}, p_{l m}\right.$, $\left.p_{l n}, p_{m n}\right\}$ is such a sixtuplet. Since there are ten $(3,3)$-partions of the indices, we have counted all the ten remaining singular planes. For brevity of notation we denote by $H_{j k}(2 \leq j<k \leq 6)$ the singular plane corresponding to the partition into $\{1, j, k\}$ and the complement. Thus we have the sixteen singular planes $H_{i}, 1 \leq i \leq 6 ; H_{j k}, 2 \leq j<k \leq 6$. In accordance with the notation for the singular points, we fix the first index as reference and put

$$
H_{0}:=H_{1} \quad H_{1 j}:=H_{j}, \quad 2 \leq j \leq 6 .
$$

Then the correspondence $q_{0} \leftrightarrow H_{0}, q_{j k} \leftrightarrow H_{j k}(l \leq j<k \leq 6)$ preserves the incidence relation between points and planes. It is thus a duality of what we call the Kummer configuration of $S$; that is the set of singular points and planes :

$$
\mathscr{K}(S):=\left\{q_{0}, q_{j k} ; H_{0}, H_{j k}\right\} .
$$

To introduce the homological counterpart of $\mathscr{K}(S)$ we observe the minimal desingularization of $S$, denoted by $\widetilde{S}$. It is obtained to be the double cover of the blowing up of $P^{2}(\boldsymbol{C})$ with center $p_{j k}(l \leq j<k \leq 6)$ branched over the proper transforms of $l_{i}(1 \leq i \leq 6)$, so we have the natural commutative diagram:

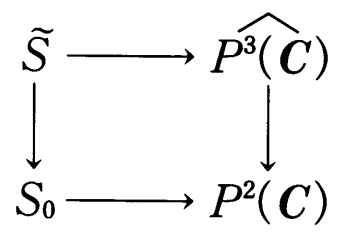

where $\widehat{P^{3}(\boldsymbol{C})}$ denotes the blowing up. We denote by $E_{0}, E_{j k}$ the exceptional divisors on $\widetilde{S}$ associated with $q_{0}, q_{j k}$. We denote further the proper transforms to $\widetilde{S}$ of the conics $H_{0} \cap S, H_{j k} \cap S$ by $C_{0}, C_{j k}$. By the same letters we also mean the homology classes of these curves, as far as the confusion is avoidable. We let $L$ stand for the class of plane sections of $S$ i. e. the natural polarization class of the quartic. The intersection behavior and the linear dependence among these classes are now summarized in the following theorem:

THEOREM 1. $E_{0}, E_{j k}$ are mutually orthogonal with respect to the intersection pairing (, ). We have also 


$$
\text { (i ) }\left\{\begin{array}{l}
(L, L)=4,\left(L, E_{0}\right)=\left(L, E_{j k}\right)=0 \\
\left(E_{0}, E_{0}\right)=\left(E_{j k}, E_{j k}\right)=-2
\end{array}\right.
$$

The expression for $C_{0}, C_{j k}$ is :

$$
\text { (ii) } \begin{cases}C_{0}=\left(L-E_{0}-\sum_{k=2}^{6} E_{1 k}\right) / 2 & \\ C_{1 j}=\left(L-E_{0}-\sum_{i=1}^{j-1} E_{i j}-\sum_{k=j+1}^{6} E_{j k}\right) / 2 & 2 \leq j \leq 6 \\ C_{j k}=\left(L-E_{1 j}-E_{1 k}-E_{j k}-E_{l m}-E_{l n}-E_{m n}\right) / 2 & 2 \leq j<k \leq 6\end{cases}
$$

where $\{l<m<n\}$ is the complementary index set to $\{1, j, k\} . C_{0}, C_{j k}$ are mutually orthogonal and we obtain the dual version of (i), (ii) by the transposition $C_{0} \leftrightarrow E_{0}, C_{j k} \leftrightarrow E_{j k}, L \leftrightarrow L^{*}$ where $L^{*}=3 L-E_{0}-\sum_{1 \leq j<k \leq 6} E_{j k}$. Since $C_{0}, C_{j k}$ are conics, we have

$$
\text { (iii) }\left(L, C_{0}\right)=\left(L, C_{j k}\right)=2 \text {. }
$$

The covering automorphism of $\widetilde{S} \rightarrow \widehat{P^{2}(\boldsymbol{C})}$ induces an automorphism of the Néron-Severi lattice of $\widetilde{S}$. We denote these automorphism by the same letter $\alpha$. The homology classes $C_{0}, C_{1 j}(2 \leq j \leq 6), E_{j k}(1 \leq j<k \leq 6)$ are invariant under $\alpha$, while the curves $E_{0}, C_{j k}(2 \leq j<k \leq 6)$ are mapped to some other curves. If we consider $L, E_{0}, E_{j k}$ as a $\boldsymbol{Q}$-base of the Néron-Severi group, the action $\alpha$ is given simply by

$$
\text { (iv) }\left\{\begin{array}{l}
\alpha(L)=3 L-4 E_{0} \\
\alpha\left(E_{0}\right)=2 L-3 E_{0}
\end{array}\right.
$$

and consequently we have

$$
\left(\text { iv }^{\prime}\right) \begin{cases}\alpha\left(C_{j k}\right)=C_{j k}+L-2 E_{0} & 2 \leq j<k \leq 6 \\ \left(E_{0}, \alpha\left(E_{0}\right)\right)=0 & \\ \left(C_{j k}, \alpha\left(C_{j k}\right)\right)=0 & 2 \leq j<k \leq 6 .\end{cases}
$$

Note that $L-E_{0}$ is stable under $\alpha$; it is the degree 2 polarization, induced from $P^{2}(\boldsymbol{C})$ by $S_{0} \rightarrow P^{2}(C)$.

\section{$\S 2$. Construction of $L\left(E_{8}\right) \oplus N$}

By the intersection behavior of rational curves described in the previous section, we can, for example, extract the following $E_{8}$-configuration:

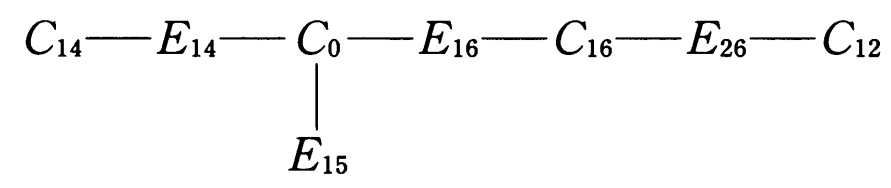

In this diagram two curves are connected as usual by an edge if and only 
if their intersection number is equal to one; other wise they are orthogonal. We put

$$
\begin{array}{llll}
r_{1}:=C_{14} & r_{2}:=E_{14} & r_{3}:=E_{15} & r_{4}:=C_{0} \\
r_{5}:=E_{16} & r_{6}:=C_{16} & r_{7}:=E_{26} & r_{8}:=C_{12}
\end{array}
$$

to be the Dynkin base for the lattice

$$
L\left(E_{8}\right):=\sum_{i=1}^{8} \boldsymbol{Z} r_{i}
$$

In order to obtain a suitable complementary Nikulin lattice to $L\left(E_{8}\right)$, we now extend (2) to the following:

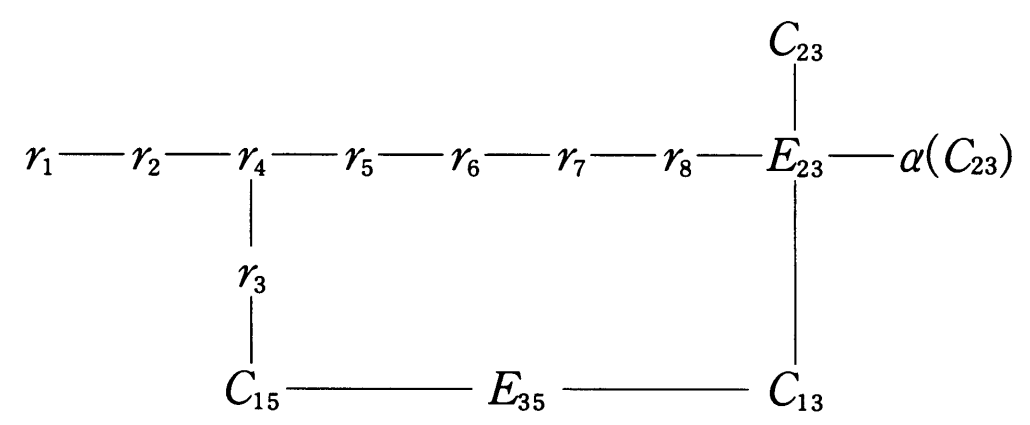

and denote by $M$ the lattice generated by $r_{1}, r_{2}, \ldots, r_{8}, E_{23}, C_{23}$ and $\alpha\left(C_{23}\right)$. $M$ is of rank 11, and it contains $L\left(E_{8}\right), C_{23}, \alpha\left(C_{23}\right)$ which are mutually orthogonal; these together generate a sublattice $M^{\prime}$ of rank 10 , so the orthogonal complement of $M^{\prime}$ in $M$ is of rank 1 , and we can choose uniquely an effective generator for it:

$$
\begin{aligned}
P:= & 16\left(L-E_{0}\right)-9 E_{12}-7 E_{13}-E_{23}-4 E_{24} \\
& -2 E_{25}-2 E_{34}-4 E_{36}-3 E_{45}-7 E_{46}-5 E_{56} .
\end{aligned}
$$

We can immediately check that $(P, P)=4$ and that the image of the $\boldsymbol{Z}$ linear form

$$
U \ni D \mapsto l_{P}(D):=(P, D) \in \boldsymbol{Z}
$$

is exactly $2 \boldsymbol{Z}$, where we denote by $U$ the lattice generated by the classes $L, E_{0}, C_{0}, E_{j k}$ and $C_{j k}(1 \leq 5<k \leq 6)$. By Diagram (3) we see that the curve $E_{35}$ is orthogonal to $L\left(E_{8}\right), C_{23}, \alpha\left(C_{23}\right), P, E_{35}$ in $U$. By checking directly we see that this is generated (over $\boldsymbol{Z}$ ) by the following five $(-2)$-classes:

$$
\begin{aligned}
& e_{1}:=\left(L-E_{0}\right)-\left(E_{12}+E_{46}\right) \\
& e_{2}:=2\left(L-E_{0}\right)-\left(E_{12}+E_{13}+E_{24}+E_{46}+E_{56}\right) \\
& e_{3}:=3\left(L-E_{0}\right)-2 E_{12}-\left(E_{13}+E_{24}+E_{36}+E_{45}+E_{46}+E_{56}\right) \\
& e_{4}:=4\left(L-E_{0}\right)-2\left(E_{12}+E_{13}+E_{46}\right)-\left(E_{24}+E_{25}+E_{36}+E_{45}+E_{56}\right)
\end{aligned}
$$




$$
\begin{aligned}
e_{5}:=5\left(L-E_{0}\right) & -3 E_{12}-2\left(E_{13}+E_{46}+E_{56}\right) \\
& -\left(E_{24}+E_{25}+E_{34}+E_{36}+E_{45}\right) .
\end{aligned}
$$

These are orthogonal to each other. They are so chosen that their intersection numbers with $L, E_{0}, E_{j k}$ etc. are non-negative. By setting

$$
e_{6}:=C_{23} \quad e_{7}:=\alpha\left(C_{23}\right) \quad e_{8}:=E_{35}
$$

we have obtained eight orthogonal $(-2)$-classes $e_{i}(1 \leq i \leq 8)$ which are orthogonal to the lattice $L\left(E_{8}\right)$. By using the expressions for $C_{j k}$ in terms of $L, E_{0}, E_{j k}$ we obtain the equality

$$
\begin{aligned}
\sum_{i=1}^{8} e_{i}=2 C_{13}+2 & \left\{8\left(L-E_{8}\right)\right. \\
& \left.-\left(5 E_{12}+3 E_{13}+2 E_{24}+E_{25}+E_{36}+2 E_{45}+4 E_{46}+3 E_{36}\right)\right\}
\end{aligned}
$$

which shows that $\sum_{i=1}^{8} e_{8}$ is divisible by 2 in the Néron-Severi group of $\widetilde{S}$. We denote the lattice generated by $e_{1}, e_{2}, \ldots, e_{8}, \sum_{i=1}^{8} e_{i} / 2$ by $N$. The question is now whether or not $N$ is primitive in the Néron-Severi group (or equivalently in $H^{2}(\widetilde{S}, \boldsymbol{Z})$ ). To answer this question we recall the lattice $U$ generated by $L, E_{0}, E_{j k}, C_{0}, C_{j k}$. $U$ is of determinant $2^{6}$.

If the Kummer quartic $S$ is generic, then $U$ coincides with the NeronSeveri lattice. In particular $U$ is primitive in that case. Since any Kummer quartic is in a smooth deformation with the generic Kummer quartic, $U$ is always primitive. Thus, if $N$ is primitive in $U$, then it is primitive in $H^{2}(\widetilde{S}, \boldsymbol{Z})$. Now, to show that $N$ is primitive in $U$, we denote its primitive hull in $U$ by $\hat{N}$. Recall also that the image of the $Z$-linear form $l_{P}$ over $U$ is $2 \boldsymbol{Z}$. We compare the two lattices $L\left(E_{8}\right) \oplus \widehat{N} \oplus \boldsymbol{Z} P \subseteq U$ where $L\left(E_{8}\right) \oplus \widehat{N}$ is exactly the kernel of $l_{P}$. Since the image of the restriction of $l_{P}$ to the former lattice is $4 \boldsymbol{Z}((P, P)=4)$ and $l_{P}(U)=2 \boldsymbol{Z}, L\left(E_{8}\right) \oplus \hat{N} \oplus \boldsymbol{Z} P$ is of index 2 in $U$ which proves that

$$
\text { determinant of } \widehat{N}=2^{6} \times 2^{2} / 4=2^{6}=\text { determinant of } N \text {. }
$$

Thus we have $\hat{N}=N$, showing the primitiveness of $N$. We have constructed an explicit primitive sublattice $L\left(E_{8}\right) \oplus N$ in the Néron-Severi group of $\widetilde{S}$; the existence of such a sublattice is guaranteed by [6]. But what is non-trivial is the following:

THEOREM 2. The sixteen (-2)-classes $r_{1}, r_{2}, \ldots, r_{8} ; e_{1}, e_{2}, \ldots, e_{8}$, which give the above $L\left(E_{8}\right) \oplus N$, are represented by non-singular rational curves on the minimal desingularization $\widetilde{S}$ if $S$ is a generic Kummer quartic. 
The representability is evident for $r_{1}, r_{2}, \ldots, r_{8}, e_{6}, e_{7}, e_{8}$. We know that the $(-2)$-cycles $e_{1}, \ldots, e_{5}$ are effective, up to sign by, Riemann-Roch. But they themselves are effective, since they have positive intersection numbers with the nef cycle $L$. The uniqueness and irreducibility of the curves representing them is then a generic condition. We now explain the geometric description of the classes $e_{1}, \ldots, e_{5}$, which gives an alternative proof of the theorem. From the expression of $e_{1}$ we see that it is represented by the proper inverse image of the line passing through $p_{12}$ and $p_{26}$ under the covering map $\widetilde{S} \rightarrow P^{2}(\boldsymbol{C})$. The class $e_{2}$ is represented by the proper inverse image of the conic passing through the five points $p_{12}, p_{13}$, $p_{24}, p_{46}, p_{56}$ among which no three are collinear. Under the genericity assumption we see that $e_{3}$ is represented by the proper inverse image of the cubic passing through $p_{13}, p_{24}, p_{36}, p_{45}, p_{46}, p_{56}$ and having a double point at $p_{12}$. The class $e_{4}$ is represented by the proper inverse image of the quartic passing through $p_{24}, p_{25}, p_{36}, p_{45}, p_{56}$ and having double points at $p_{12}$, $p_{13}, p_{46}$. The class $e_{5}$ is represented by the proper inverse image of the quintic passing through $p_{24}, p_{25}, p_{34}, p_{36}, p_{45}$, having double points at $p_{13}, p_{46}$, $p_{56}$ and a triple point at $p_{12}$. By counting the number of parameters for curves of the corresponding degree and the number of linear conditions which they should satisfy, we see that, for each case, there is at least one curve which fulfills the above requirements. The genericity of $S$ is needed only for the uniqueness and irreducibility of those curves and the smoothness of their proper transforms. (This should be regarded as the definition of genericity.)

\section{$\S 3 . \quad$ A birational geometry}

Recall that $\widehat{P^{2}(\boldsymbol{C})}$ is the blowing up of $P^{2}(\boldsymbol{C})$ whose center consists of the fifteen intersection points $p_{j k}(1 \leq j<k \leq 6)$ of the six lines $l_{i}(1 \leq i \leq$ 6). The minimal desingularization $\widetilde{S}$ is described as the double cover of $\widehat{P^{2}(\boldsymbol{C})}$ branched over the proper transforms $\hat{l}_{i}$ of $l_{i}(1 \leq i \leq 6)$. $\quad S$ was assumed to be so generic that the five $(-2)$-classes $e_{1}, e_{2}, \ldots, e_{5}$ are represented by non-singular rational curves on $\widetilde{S}$, which we denote now by $D_{1}, D_{2}, \ldots, D_{5}$. Each $D_{i}$ is the proper inverse image of some exceptional curve of the first kind on $\widehat{P^{2}(\boldsymbol{C})}$. We denote this curve by $D_{i}^{\prime}$. For $E_{j k}$ $(1 \leq j<k \leq 6)$ their images on $\widehat{P^{2}(\boldsymbol{C})}$, which we denote similarly by $E_{j k}^{\prime}$, are also exceptional curves of the first kind; they are the exceptional sets associated with $p_{j k}$. The curves $C_{0}, C_{1 j}(2 \leq j \leq 6)$ are exactly the branch- 
ing locus on $\widetilde{S}$; they are identified with the $(-4)$-curves $\hat{l}_{1}, \hat{l}_{2}, \ldots, \hat{l}_{6}$. The ten curves $C_{j k}(2 \leq j<k \leq 6)$ are disjoint from the branching locus, so $C_{j k}$ and $\alpha\left(C_{j k}\right)$ are disjoint, and they form the proper inverse image of the image $C_{j k}^{\prime}$ on $P^{2}(\widehat{C}) ; C_{j k}^{\prime}$ is the proper transform of the conic passing through $p_{1 j}, p_{1 k}, p_{j k}, p_{l m}, p_{l n}, p_{m n}(\{1<j<k, l<m<n\}=\{1,2, \ldots, 6\})$. The ten curves $C_{j k}^{\prime}$ are $(-2)$-curves. Thus, by applying the map $\widetilde{S} \rightarrow \widehat{P^{2}(\boldsymbol{C})}$ to Diagram (3) and adding $D_{i}^{\prime}(1 \leq i \leq 5)$ we obtain

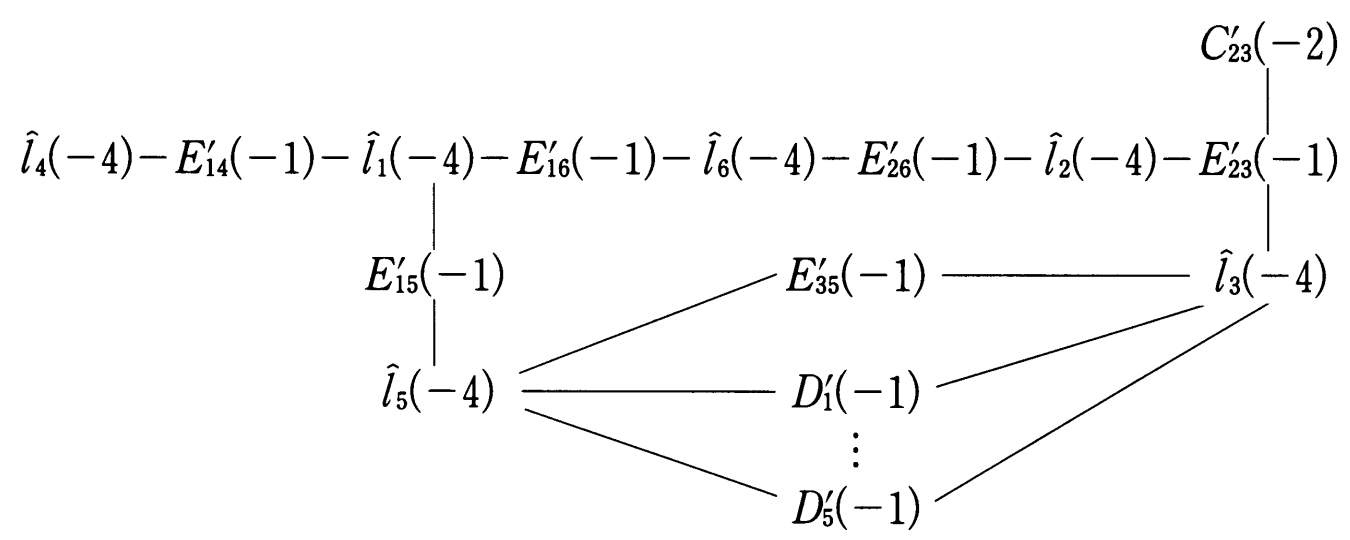

where the number in a parenthesis denotes the self-intersection number of the corresponding curve. We see now that we can step by step blow down the fifteen curves $E_{14}^{\prime}, E_{15}^{\prime}, E_{16}^{\prime}, E_{26}^{\prime}, E_{23}^{\prime}, E_{35}^{\prime}, D_{1}^{\prime}, \ldots, D_{5}^{\prime}, \hat{l}_{1}, \hat{l}_{4}, \hat{l}_{6}, C_{23}^{\prime}$ to reach a non-singular rational surface of the Euler number 3 . This is therefore another projective plane, which we denote by $P^{2}(C)^{\prime}$ to distinguish it from the original $P^{2}(\boldsymbol{C})$. The images on $P^{2}(\boldsymbol{C})^{\prime}$ of the curves $\hat{l}_{5}$, $\hat{l}_{2}, \hat{l}_{3}$ are now a cubic with a cusp, the line intersecting the cubic only at the cusp and a conic touching the line at one point. These three curves form a reducible sextic whose singular points are the cusp, the contact point of the line and the conic, and the six intersection points of the conic and the cubic. The cusp is a rational double point of Type $E_{7}$ and the contact point is of Type $A_{3}$; the other six singular points are of Type $A_{1}$ (nodes in the classical terminology). We see the corresponding $E_{7^{-}}$ configuration formed by $r_{1}, r_{2}, \ldots, r_{7}$ and the $A_{3}$-configuration formed by $C_{23}=e_{6}, E_{23}$ and $\alpha\left(C_{23}\right)=e_{7}$. The six $(-2)$-curves are naturally $D_{i}=e_{i}(1$ $\leq i \leq 5)$ and $E_{35}=e_{8}$. Thus we can easily read up the primitive lattice $L\left(E_{8}\right) \oplus N$ on this sextic double plane. We know that this is a Kummer surface theoretically, as was mentioned several times. In fact, the motivation to this work came out of the attempt to find sixteen disjoint nonsingular curves on this sextic double plane.

To close this note, we remark that there is an interesting theoretical Kummer surface for which sixteen disjoint $(-2)$-curves have not been 
found yet. The Néron-Severi lattice is of rank 18 and of determinant -80 for it. It contains $N$ and a lattice of rank 10 described by a double hexagonal diagram; the second lattice is orthogonal to $N$ and it contains $L\left(E_{8}\right)$. See [7] for the detailed description of the surface.

Acknowledgment. I thank the referee for the revision of the proof of Theorem 2.

\section{References}

[1] BORChARDT, C. W., Über die Darstellung der Kummerschen Fläche vierter Ordnung mit sechzehn Knotenpunkten durch die Göpelsche biquadratische Relation zwischen vier Thetafunktionen mit zwei Variablen, J. für reine angew. Math., 83 (1977), 234-250.

[2] BRIESKORN, E., Über die Auflösung gewisser Singularitäten von holomorphen Abbildungen, Math. Ann., 166 (1966), 76-102.

[ 3 ] BRIESKORN, E., Die Auflösung der rationalen Singularitäten holomorpher Abbildungen, Math. Ann., 178 (1968), 255-270.

[4] CaYley, A., The double $\Theta$-functions in connexion with a 16 -nodal quartic surface, J. für reine angew. Math., 83 (1877), 210-219.

[ 5 ] Humbert, G., Oeuvres de George Humbert II, Gauthier-Villars, Paris, 1929.

[ 6 ] MORRISON, D. R., On $K 3$ surfaces with large Picard number, Invent. math., 75 (1984), 105-121.

[ 7 ] NARUKI, I., Double hexagon on a $K 3$ surface, to appear in Hokkaido Math. J.

[ 8 ] WEBER, H., Über die Kummersche Fläche vierter Ordnung und ihre Beziehung zu den Thetafunktionen mit zwei Veränderlichen, J. für reine angew. Math., 84 (1878).

Research Institute for Mathematical Sciences, Kyoto University Kitashirakawa, Sakyo-ku, Kyoto 606 Japan 